\title{
Track test monitoring system using a multipurpose experimental train
}

\author{
H. Matsuda ${ }^{1}$, M. Takikawa ${ }^{1}$, T. Nanmoku ${ }^{2} \&$ E. Yazawa ${ }^{2}$ \\ ${ }^{1}$ East Japan Railway Company, Japan \\ ${ }^{2}$ Railway Technical Research Institute, Japan
}

\begin{abstract}
In order to inspect the condition of tracks, which support railway cars, track irregularity is measured four times a year along conventional lines by track inspection cars, called "East-i". Track irregularity and the track materials that cannot be inspected with the track inspection car are regularly inspected by track patrolling on foot and/or using hand-held type inspection instruments. With the purpose of reducing track patrol labor and maintenance costs, as well as to improve inspection quality, we have been developing a track monitoring system that is installed on a commercial car and monitors the track with greater frequency. This paper provides the outline for a track measurement device that uses the inertial mid-chord offset method and a track-material monitoring device, both of which have been developed to monitor the track, and presents the results of installing these devices on the multipurpose experimental train (called "MUETrain") on conventional lines.
\end{abstract}

Keywords: track monitoring system, inertial mid-chord offset method, trackmaterial monitoring device, multipurpose experimental train.

\section{Introduction}

Tracks are composed of rails, sleepers, ballast, and other materials. Track irregularity advances and track material deteriorate as a result of repeated car load and severe environmental conditions. So, it is important to monitor, inspect and understand track irregularity and the degree and process of deterioration of track materials.

In order to inspect track conditions, track irregularity is measured four times a year along conventional lines in East Japan Railway Company by track 
inspection cars called "East-i". Track irregularity and the track materials that cannot be inspected with the track inspection car are regularly inspected by track patrolling on foot and/or using hand-held type inspection instruments. However, this is time-consuming and labor-intensive when inspecting over long distances. So, an efficient inspection method is needed to reduce time and cost. Moreover, it is necessary to maintain a continuous awareness of track conditions, and to know about potential problems to improve efficiency of inspection.

We have been developing a track monitoring system, which is installed on a commercial car and monitors the track with greater frequency. This paper provides the outline for a track measurement device that uses the inertial midchord offset method (hereinafter referred to as IMOM) and a track-material monitoring device, both of which have been developed to monitor the track, and presents the results of installing these devices on the multipurpose experimental train called "MUE-Train" on conventional lines. MUE-Train is a test train converted from former Keihin-Tohoku line 209 series cars to be used to test and develop new technology for use on future trains.

\section{Track measurement device using the IMOM}

\subsection{Outline of a track measurement device using the IMOM}

The track measurement device using the IMOM is composed only of a sensor box under the car-body and a small equipment box on board which together collect data which can measure track irregularity (e.g., gauge, alignment, longitudinal level, cross level, twist) [1]. The current track inspection car is a complex and large device with a two-bogie measurement system and rail displacement sensors mounted on all the axle boxes. Consequently, it cannot be installed on a commercially used car. The track measurement device using IMOM was selected for its compact size and ease in mounting.

With this device we measure the track irregularity using IMOM. The displacement of the device is obtained by measuring the acceleration of the car body and integrating it as well as using two axis rail displacement sensors to measure relative displacement between the device and the rail, measured by laser. It is possible to downsize and lighten the device, and reduce its cost from the current one that occupies a single car since track irregularity is determined by measurement at a single point. Moreover, the current track inspection car involves the dismantling of related parts installed together with the axle box when inspecting it in the car factory and the reassembly after the inspection is finished. However, dismantling and the assembly operations became unnecessary for the new device as it is external.

\subsection{Development of a body-mounted device using the IMOM}

This device has already been used, mounted on a bogie, by Kyushu Shinkansen [2]. However, it would be difficult to mount on certain bogies due to dimensional constraints. So, we developed a body-mountable device with fewer restrictions to 


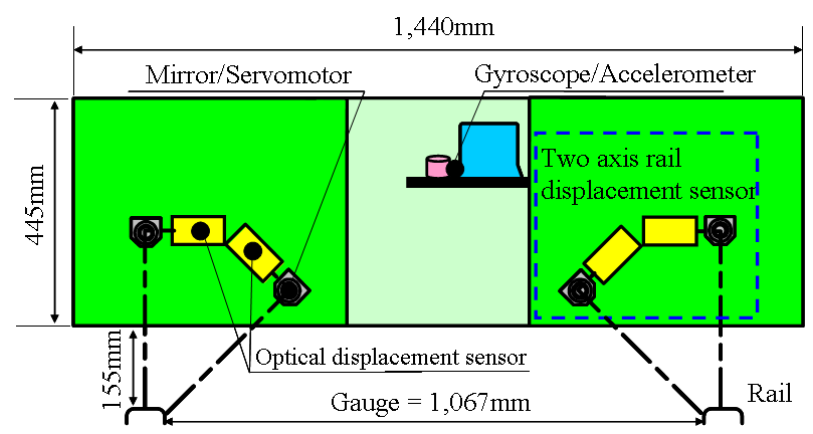

Figure 1: Composition of a body-mounted device.

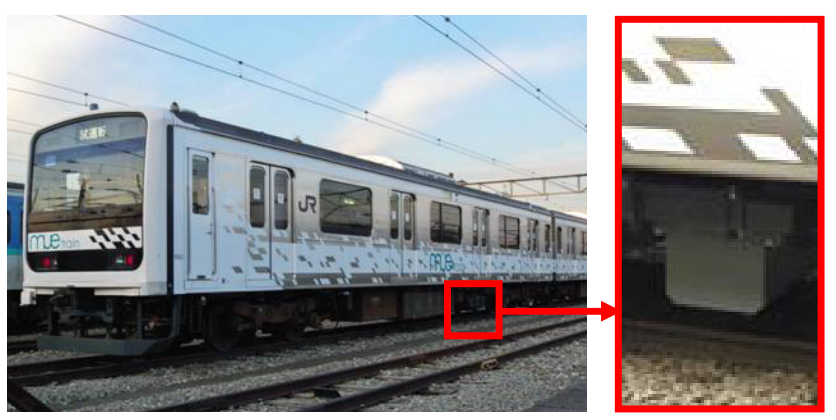

Figure 2: $\quad$ MUE-Train and device.

mounting positions (Figure.1). In this case, the device is somewhat away from the rail, thus necessitating a wider measurable range, resulting in the possibility that foreign objects might also be caught within its range. It is thus important to make improvements so that the object being measured can be properly ascertained.

\subsection{Outline of the test measurement}

We have been implementing running tests of the MUE-Train mounted with this device in the metropolitan area since January 2009 to check its measurement precision and durability. Figure 2 shows the installation of MUE-Train and the device. MUE-Train is a 7-train set, and this device is mounted as shown in Figure 2 near the bogie on the car 6 side of the car 7. The total mileage was about $12,000 \mathrm{~km}$ as of the end of April in 2010. Up to now, no trouble has occurred with this device, and it does its job well.

\subsection{Reproducibility of repeated measurement}

Accuracy in reproducibility of repeated measurements along the same section has been verified. Figure 3 shows the $10 \mathrm{~m}$-chord longitudinal level irregularity, 
while Figure 4 shows the $10 \mathrm{~m}$-chord alignment irregularity. The same section was measured seven times along the same section about every 40 days. " $\sigma$ " is the standard deviation of the reproducible error in a section concerned when based on the waveform of the highest rung. The standard deviation of the reproducible error of repeated measures is intended to be smaller than or equal to $0.3 \mathrm{~mm}$ for Shinkansen, and for conventional lines smaller than or equal to $0.5 \mathrm{~mm}$.

As seen in Figure 3,10 m-chord longitudinal level irregularity reproducible error measurement precision proves to be under $0.3 \mathrm{~mm}$ in all cases. Moreover, velocity dependency is not seen. Next, as seen in Figure 4, the reproducible error of $10 \mathrm{~m}$-chord alignment irregularity is $0.32 \mathrm{~mm}$ maximum, a level of accuracy sufficient for practical use along conventional lines. Velocity dependency is also not seen in $10 \mathrm{~m}$-chord alignment irregularity. The accuracy of the optical-fiber gyroscope greatly influences the accuracy of alignment irregularity as shown in a

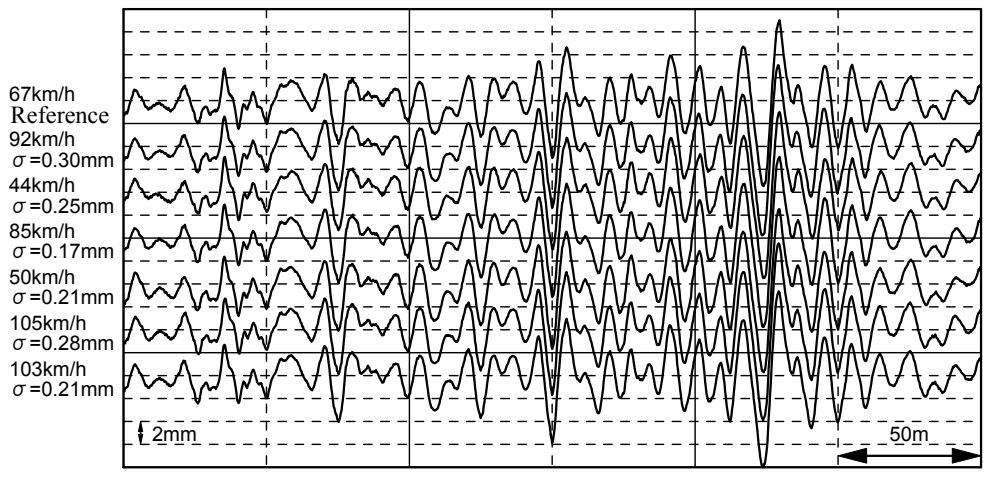

Figure 3: Reproducibility of $10 \mathrm{~m}$-chord longitudinal level irregularity.

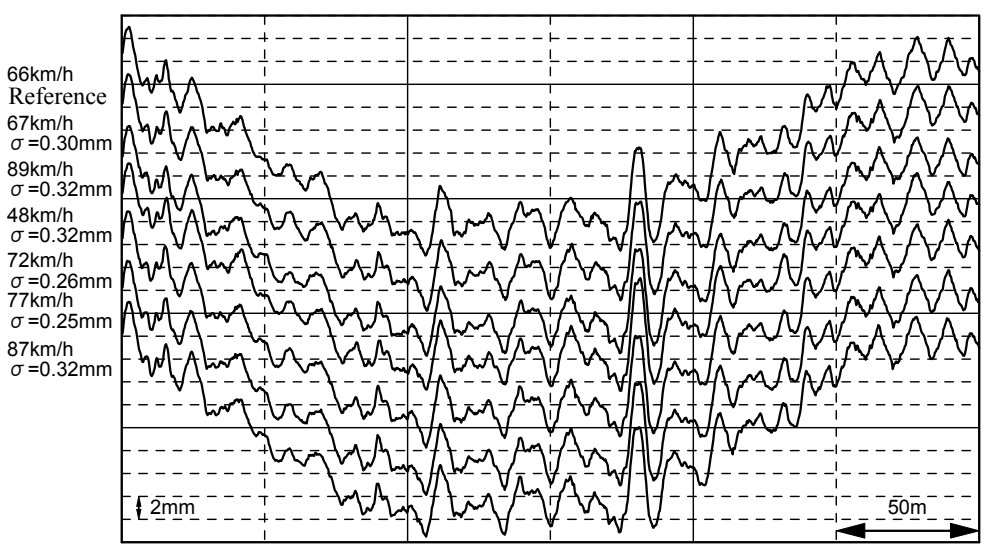

Figure 4: Reproducibility of $10 \mathrm{~m}$-chord alignment irregularity. 
curve section of this device. Therefore, further accuracy improvement can be expected by additionally adopting a gyroscope of higher accuracy.

\subsection{Consistency with the current track inspection car}

By comparing the results of measurement using this device and those of the current inspection car, data integrity was verified. Figure 5 (upper) shows $10 \mathrm{~m}$ chord longitudinal level irregularity, while Figure 5 (lower) shows the $10 \mathrm{~m}$ chord alignment irregularity. The results from this device show the value measured three days after the measurement of that of the track inspection car, the measurement speed of both being about $60 \mathrm{~km} / \mathrm{h}$.

In $10 \mathrm{~m}$-chord longitudinal level irregularity, the waveform of this device is identical to that of the track inspection car. In $10 \mathrm{~m}$-chord alignment irregularity, both shape and amplitude correspond roughly to each other's waveforms, though small differences in detail can be seen due to difference of detection method and the rail side positioning. It one can see that in both longitudinal level and in alignment, results are consistent with the current track inspection car.

\section{Track material monitoring device}

\subsection{Outline of track material monitoring device}

The purpose of the track material monitoring device is to take images and automatically detect abnormalities: missing fishplate bolts, loosened rail fastening, shifted rail pads, metal flow at glued-insulation rail, etc (Figure 6).

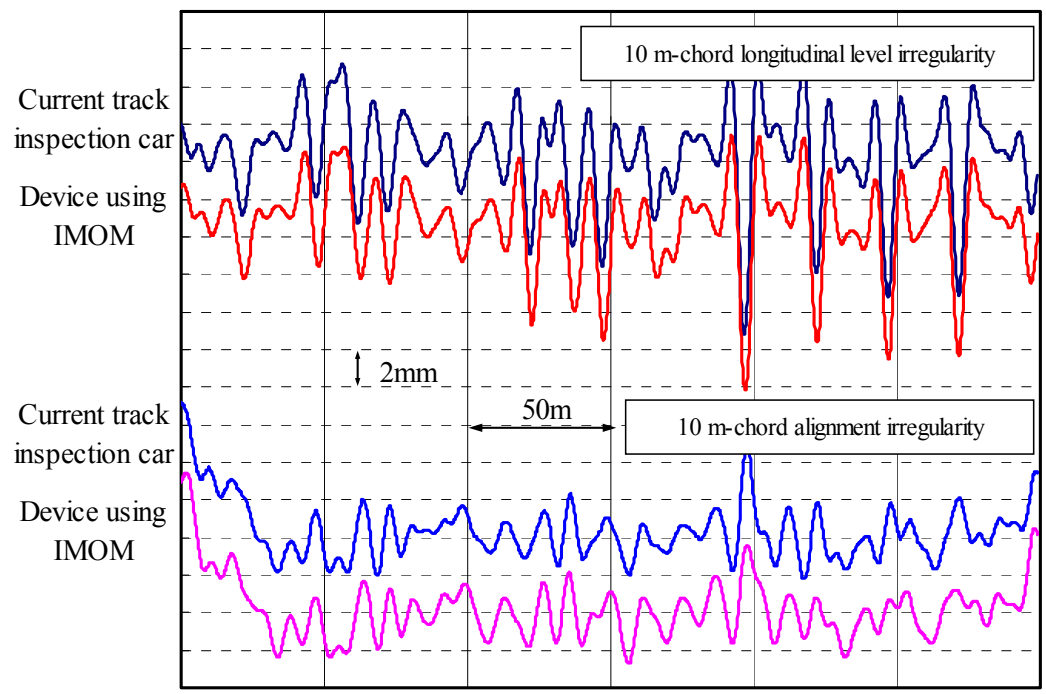

Figure 5: Comparison with current track inspection car. 


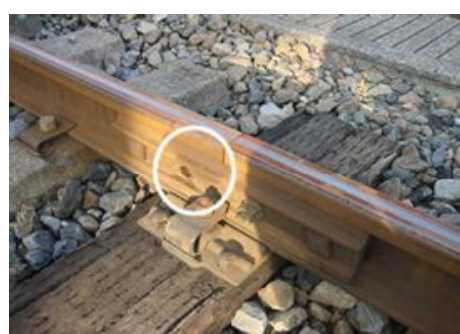

(a) Missing fishplate bolt

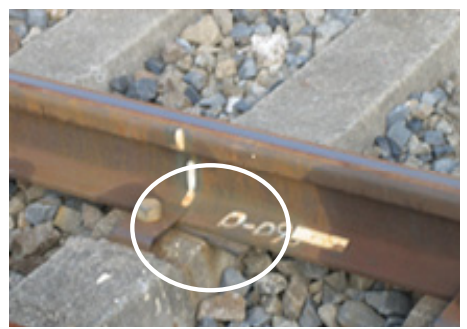

(c) Shifted rail pad

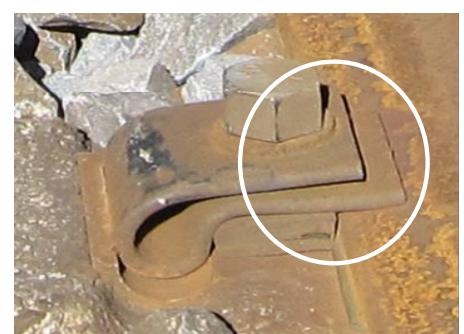

(b) Loosened rail fastening

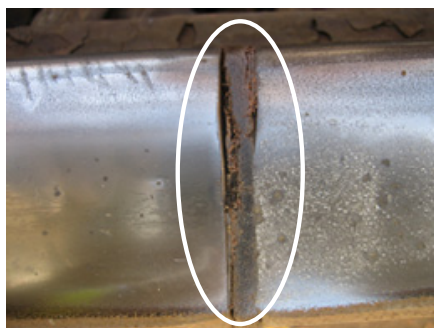

(d) Metal flow at gluedinsulation rail

Figure 6: Examples of abnormality of track material.

This device, which takes images of the track material with a line sensor camera, has already been put to practical use both domestically and abroad. However, not many cases have been seen regarding its function of automatically judging abnormality in track material. If it could photograph abnormalities with greater frequency and could judge automatically, the reliability of the track equipment would improve and it would reduce the labor of track patrol.

The device is composed of two cameras: 1) a line sensor camera for taking pictures of the track material: and 2) a device for acquiring altitude information (three-dimensional image) in the vicinity of the rail. As part of this development, we plan to utilize altitudinal information to conduct automatic judgments on abnormalities.

\subsection{Outline of running tests}

The track material monitoring device has been installed on the MUE-Train, and data collected along the Tohoku and Nikko lines since January 2009. Figure 7 shows the device installed on the MUE-Train. During this time, the device was installed on only one side of the rail. 


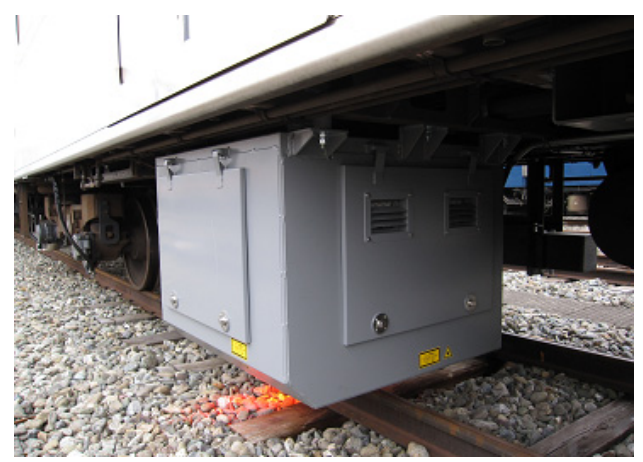

Figure 7: Track material monitoring device installed on the MUE-Train.

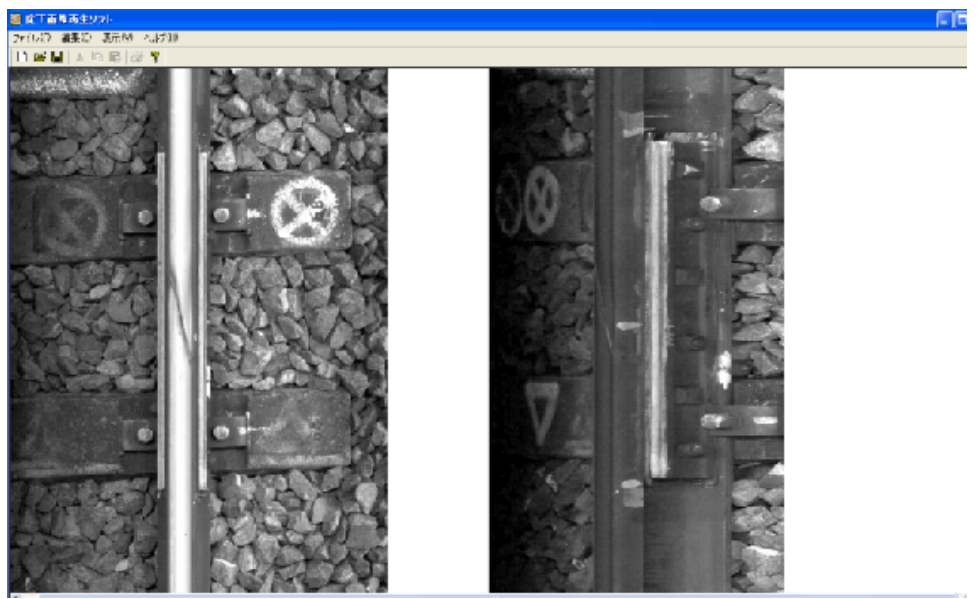

Figure 8: $\quad$ Examples of photographic image.

\subsection{Test results}

Figure 8 shows a photographic image of track material. Figure 9 shows a three dimensional image of track material around the rail. In this figure, higher areas are shown as white, while lower areas are black. However, the top of rail was not considered at this time. The cross section along the line is showed on the screen below. In this test, the altitude information in the vicinity of the rail were measured to an accuracy of $1 \mathrm{~mm}$ up to $110 \mathrm{~km} / \mathrm{h}$. Tightness of the rail fastening can be evaluated by means of measuring the height of the upper spring crip with respect to the rail foot. 


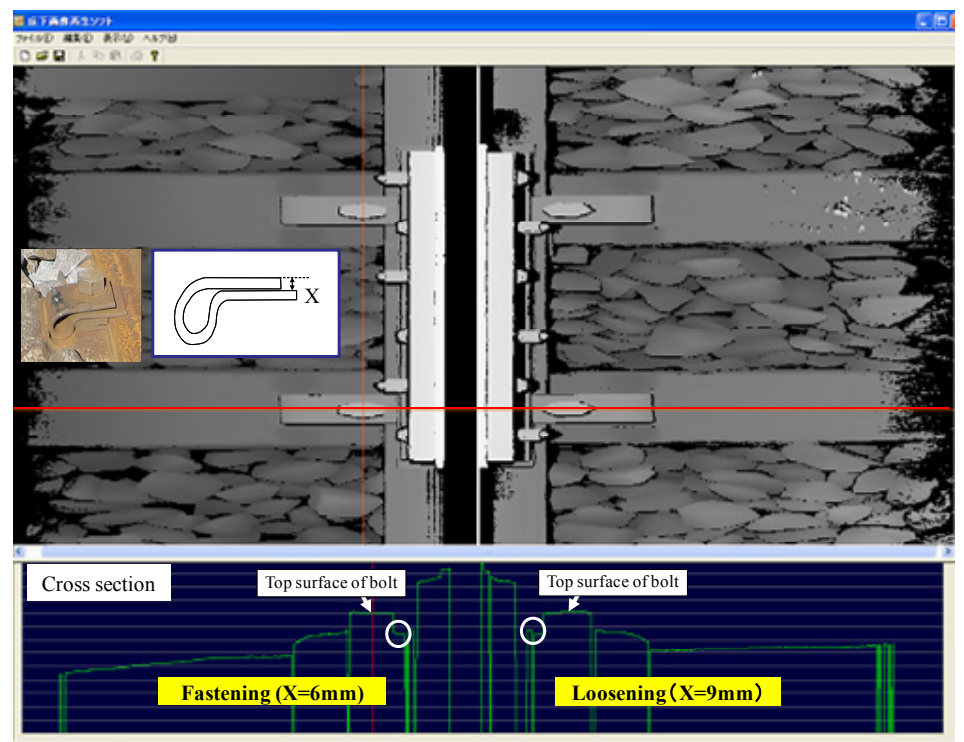

Figure 9: $\quad$ Examples of three-dimensional image of track material.

\section{Conclusion}

For the track material monitoring device, we obtained measurements to an accuracy of $1 \mathrm{~mm}$ for altitude information in the vicinity of the rail up to 110 $\mathrm{km} / \mathrm{h}$. At present, an automatic judgment function is not included in this device, but with the accumulation and verification of data, it is to be included in the future. On the other hand, the track measurement device using IMOM achieved the high level of accuracy required for practical use. Moreover, it will continue to be put to use and its durability will be tested. Eventually, the on-board portion of the device should be made more compact for efficient use on commercial cars in the future.

\section{References}

[1] Yazawa, E. \& Takeshita, K., Development of Measurement Device of Track Irregularity using Inertial Mid-chord Offset Method. QR of RTRI, Vol.43, No.3, pp.125-130, 2002

[2] Moritaka, H., Matsumoto, T. \& Yazawa, E., Measurement of Track Irregularity using Kyushu Shinkansen Cars. Shinsenro, Vol.63, No.12, pp.26-28, 2009 (in Japanese) 